\title{
INTERACTIVE MULTIVIEW IMAGE CODING
}

\author{
Andriy Gelman ${ }^{1}$, Pier Luigi Dragotti ${ }^{1}$, Vladan Velisavljevic ${ }^{2}$ \\ ${ }^{1}$ Communications and Signal Processing Group, Imperial College, London, UK \\ ${ }^{2}$ Deutsche Telekom Laboratories, Technische Universität Berlin, Germany
}

\begin{abstract}
We propose a novel multiview compression method for multiview images. The algorithm supports random access for interactive applications and has low storage requirements. The fundamental component of the method is the layer-based representation, which partitions the data set into redundant layers characterized by a constant depth value. We exploit the redundant property of each layer and remove the side information uncertainty using Distributed Source Coding (DSC) principles. In comparison to JPEG2000, our algorithm achieves a PSNR improvement of 1.6dB. Furthermore, we present a rate-distortion (RD) analysis which demonstrates that the proposed algorithm can achieve a better performance in comparison to independent coding.
\end{abstract}

\section{INTRODUCTION}

In recent years, Image Based Rendering (IBR) has been proposed as an alternative to the traditional rendering algorithms. The approach has a lower computational complexity and achieves photorealistic results by interpolating the novel viewpoints from existing data. To obtain artifact-free results, however, the scene must be sampled with a large number of cameras. These images are either transmitted or stored, which means efficient compression is an essential part of IBR systems [1].

The majority of the compression literature has focused on hierarchical prediction [2] or subband coding [3, 4]. Although these algorithms achieve high compression, they have limited random access. These techniques are, therefore, not suitable for an interactive setting, where the images are stored at server and transmitted to the remote users on request. The key point is that the viewing trajectory is unknown prior to encoding.

A number of techniques have been proposed which achieve high compression and still maintain random access. For example, in [5] the authors propose storing multiple representations of an image for a set of possible predictions to reduce the transmission rate and eliminate drift. This method, however, requires high storage requirements at the server. A different approach $[6,7]$ has been to use DSC principles to reduce the storage size and eliminate the side information uncertainty.

In this paper we propose a novel multiview image coding method with random access and low storage requirements at the server. The fundamental component of the algorithm is the layerbased representation [8], which partitions the data set into layers each modeled by a constant depth plane. The redundancy of each layer is exploited using DSC principles, which also eliminates side information mismatch at the user. Our algorithm is designed to be encoded once at the server and decoded multiple times, where the quality of the decoded images is controlled by setting a Lagrangian parameter $\lambda$. Additionally, the algorithm is complemented with a model which demonstrates that the approach can achieve a better RD performance than independent coding of images.

The outline of this paper is as follows. Multiview data structure and the layer-based representation are reviewed next. In Section 3 we present the proposed algorithm and in Section 4 discuss its RD performance. The results are presented in Section 5 and the paper is concluded in Section 6.

\section{REVIEW OF MULTIVIEW IMAGE REPRESENTATION}

In this section, we analyze the redundancy of multiview images and review the layer-based representation. For clarity, we simplify the setup to a 1D array of uniformly spaced cameras perpendicular to the baseline, also known as an EPI [1]. This type of data set is parameterized as:

$$
I=P_{3}\left(x, y, V_{x}\right),
$$

where $I$ is the pixel intensity, $(x, y)$ are the spatial coordinates of the image and $V_{x}$ is the camera location.

\subsection{Multiview image data structure and redundancy}

Although a huge amount of multiview data is required to achieve artifact-free rendering, this data set is highly redundant. Within each image, neighboring light rays are likely to originate from the same object and, therefore, they contribute to the intra-frame correlation. In addition, due to the parallax, an object appears at different pixel locations $x$ and $x^{\prime}$ seen from different viewpoint coordinates (frames) $V_{x}$ and $V_{x}^{\prime}$, thus contributing to the inter-frame correlation (see Fig. 1). Assuming the scene is Lambertian and has no occlusions, this shift in pixel locations (disparity) $\Delta x=x-x^{\prime}$ can be represented as a function of the corresponding viewpoint coordinates, depth $Z$ of the object and focal length $f$, that is,

$$
\Delta x=\frac{f\left(V_{x}^{\prime}-V_{x}\right)}{Z} .
$$

The obtained relation between the viewpoint and spatial coordinates is commonly illustrated as a set of EPI lines. An example is shown in Fig. 2(a), where points in the 3D space are projected onto lines with slopes inversely proportional to the depth. Notice that such a set of EPI lines is highly correlated.

\subsection{Layer-Based Representation}

The concept of the layer-based representation is to partition the data into layers, where each layer is modeled by a constant depth plane and has a smaller depth variation than the original scene.

Extraction of layers from a general 3D scene is a non-trivial task. Here, we use a variation of the level-set segmentation algorithm which was proposed in [8]. An advantage of this unsupervised 


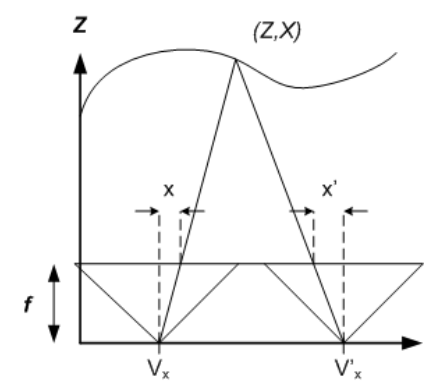

Fig. 1. Horizontal parallax model used to evaluate the pixel disparity.

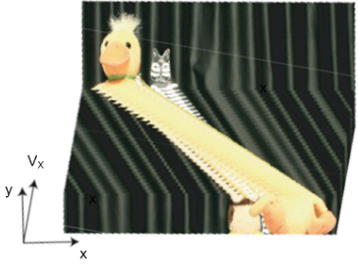

(a) Multiview image crosssection. The data can be analyzed as a set of EPI lines with varying gradients

Fig. 2. Multiview image data set cross-section and the extracted layers.

method is that it can be extended to an arbitrary number of dimensions. Furthermore, using a semi-parametric methodology, the algorithm efficiently handles occlusions, which is an important property for the subsequent compression algorithm.

Fig. 2(b) illustrates the extracted layers from the dataset in Fig. 2(a). It can be observed that each layer preserves the linear structure corresponding to an object location in a $3 \mathrm{D}$ space.

\section{PROPOSED ALGORITHM}

In this section we propose our novel approach to encoding multiview images. First, we describe the general overview of the algorithm and, then, we outline the RD optimization.

\subsection{Algorithm Overview}

The concept of the proposed algorithm is to substitute the inter-view transform with DSC coding. This property allows the decoder to correctly reconstruct the transmitted data given any side information available in the cache of the user. The DSC ideas are applied to each layer independently in the spatial transform domain. We note that the redundant properties of the layers reduce the number of data bits which must be transmitted, thus providing a bit-rate saving in comparison to independent encoding of the images. Next, we outline the encoding process for one layer, which can be generalized to the complete dataset.

Consider a layer from the 'Animal Farm' dataset shown in Fig. 3(a). Initially, a preprocessing step is applied where the occluded

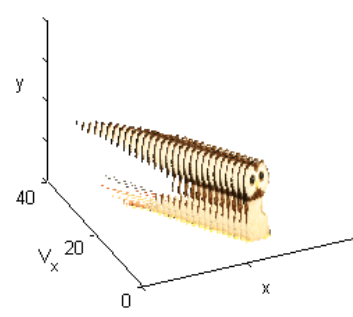

(a) Original owl layer

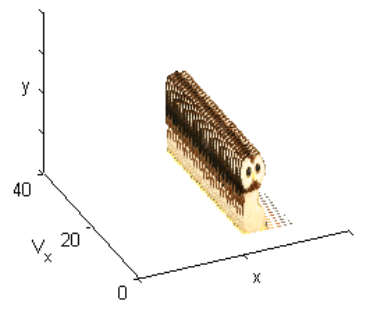

(b) Owl layer with preprocessing
Fig. 3. Interpolation of the extracted layers. (a) Extracted layers might have discontinuities in the EPI lines due to the occlusions. (b) The values in the EPI lines are interpolated using the mean of the non-occluded pixels and each image is disparity compensated onto a common view.

regions are interpolated using the mean along the EPI lines and each image is disparity compensated onto a common view. The obtained layer is shown in Fig. 3(b). Note that the layer contour in each image is constant. This boundary is losslessly encoded and transmitted along with the disparity.

In the following step, we reduce the intra-view redundancy by applying a 9/7 Discrete Wavelet Transform (DWT) to each image. We use a shape-adaptive implementation as proposed in [9] to remove the boundary effects associated with the irregular contour. Then, the resulting DWT subbands from each image are quantized using the same step size chosen in a similar approach to [4]. The low-pass transform coefficients from three images are illustrated in Fig. 4. Observe that the subbands are correlated across the views, which is exploited by the DSC algorithm in the following stage.

Recall that the cache of the remote user may contain DWT blocks from any image as side information. Our approach is to use DSC principles to remove the side information uncertainty. Consider the following model:

$$
y=x+n,
$$

where $y$ is the transform coefficient requested by the user, $x$ is the side information available in the cache and $n$ is the residual signal. Recall that $y$ can be correctly reconstructed transmitting at least $\left\lceil\log _{2}(2 n)+1\right\rceil$ least significant bits (LSB) from $y$. To encode a sequence of blocks shown in Fig. 4, we take the worst case scenario, where any image can be used as side information. For example, the transform sequence $\{55,51,53\}$ requires $\left\lceil\log _{2} 8+1\right\rceil=4 \mathrm{LSB}$ to correctly reconstruct the data. The analysis to evaluate the number of LSB required for transmission is implemented at the server prior to encoding.

The server subsequently encodes the data using a bit-plane context adaptive arithmetic coder to attain rates close to the entropy of the source. The number of retained LSB is also encoded and transmitted with the data. This information is stored by the user for future reference. Note that the number of retained LSB provides a bit-plane significance map [10], which is further exploited by the entropy coder to reduce the encoding rate.

\subsection{Transmission and RD Optimization}

The data blocks are transmitted according to a Lagrangian parameter $\lambda$ chosen by the remote user. For each residual block, we choose a transmission mode which minimizes the RD cost

$$
D_{i}+\lambda R_{i}
$$




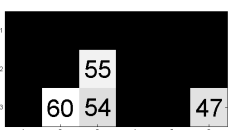

(a)

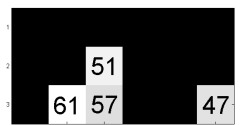

(b)

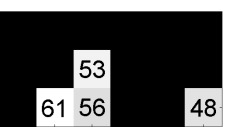

(c)
Fig. 4. Quantized low-pass subbands from three images. Observe that the blocks are correlated across the views.

where $D_{i}$ is the distortion and $R_{i}$ is the transmission cost of the $i$-th mode. These values are evaluated off-line and stored at the server.

The blocks have three different modes. These are 'skip', 'skip2 ' and 'lsb'. The first skip mode sets the data to zero and the second uses the side information in the cache as a prediction. The 'lsb' mode, however, transmits the residual data to correctly reconstruct the DWT block.

\section{THEORETICAL MODELING}

In this section we present a theoretical model of our encoding scheme. First, we present a synthetic data model which well approximates real multiview images, then evaluate the RD relation of the independent and the proposed DSC algorithm for this class of signals.

\subsection{Data Modeling}

\subsection{1. $2 D \alpha$-Lipschitz model}

We model the layer images using a globally smooth 2D $\alpha$-Lipschitz function $f_{\alpha}(x, y)$, which satisfies the following condition:

$$
\left|f_{\alpha}\left(x_{1}, y_{1}\right)-f_{\alpha}\left(x_{2}, y_{2}\right)\right| \leq K\left(\left|x_{1}-x_{2}\right|^{2}+\left|y_{1}-y_{2}\right|^{2}\right)^{\alpha / 2},
$$

where $K>0$. Transforming the signal using a 2D wavelet having at least $\lfloor\alpha+1\rfloor$ vanishing moments yields wavelet coefficients with the following decay [11]:

$$
\left|d_{j, n}\right| \leq A 2^{-j(\alpha+1)},
$$

where $j$ is the wavelet scale and constant $A>0$. A linear compression scheme based on (6) can be designed by appropriately choosing constant quantization step size across all the subbands [11]. It can be shown that a high bit-rate assumption of the compression algorithm yields the following RD function:

$$
D(R) \leq c R^{-\alpha},
$$

where $R$ is the total number of bits allocated to encoding the signal and constant $c>0$.

\subsubsection{Contour Model}

In practice, the layers are outlined by a segmentation and are therefore not globally $\alpha$-Lipschitz smooth. To obtain the decay in (7), we transmit the contours and encode the texture using a shape adaptive scheme. We model the contour of the texture as piecewise linear curve having $V$ vertices. The RD due to quantizing the location of the vertices can be upper bounded as [4]:

$$
D(R) \leq A^{2} T^{2} V 2^{-R / 2 V},
$$

where $A$ is the maximal magnitude of the texture and $T$ is the maximal length of a side of the bounding box.

\subsubsection{Multiview Image Model}

Using the analysis in Section 2.1, the layer images are modeled as a shifted version of the first view and a 2D $\alpha$-Lipschitz error term. The error term corresponds to either lighting changes, layer extraction errors or non-Lambertian surfaces.

$$
f_{i}(x, y)=f_{1}(x-(i-1) \Delta x, y)+\epsilon_{\alpha}^{i}(x, y),
$$

where $\Delta x$ is the layer disparity defined in (2) and $i$ is the image location.

\subsection{Independent Encoding}

In the case of independent encoding, the 2D $\alpha$-Lipschitz signal and the layer contour are separately encoded from each view. Using (7) and (8), the total distortion due to encoding the texture and the contour is bounded as:

$$
\mathbf{D}_{\text {ind }}\left(\mathbf{R}_{\mathbf{t}}\right) \leq \sum_{i=1}^{N} c_{i}\left(R_{x}^{i}\right)^{-\alpha}+N A^{2} T^{2} V 2^{-R_{v} / 2 V},
$$

where $R_{x}^{i}$ and $R_{v}$ is the rate allocated to the $\alpha$-Lipschitz texture in the $i$-th view and the contour encoding rate in each image, respectively and $N$ is the total number of views. The total bit-rate can be shown to be:

$$
\mathbf{R}_{\mathbf{t}}=\sum_{i=1}^{N} R_{x}^{i}+N R_{v}
$$

The correct rate allocation which minimizes the distortion for a total bit budget can be solved using Lagrangian multipliers. A high rate analysis yields:

$$
R_{x}^{i} \approx \mathbf{R}_{\mathbf{t}}\left(\sum_{l=1}^{N}\left(\frac{c_{l}}{c_{i}}\right)^{\frac{1}{\alpha+1}}\right)^{-1}
$$

and

$$
R_{v}=2 V \log _{2}\left(\frac{A^{2} T^{2} \ln (2)}{2 \alpha c_{1}}\right)+2 V(\alpha+1) \log _{2} R_{x}^{1} .
$$

The minimized RD function in terms of the total rate can be obtained by substituting (12) and (13) into (10).

\subsection{Proposed Algorithm}

Using (9) we note that the wavelet coefficients in the residual frames can described using:

$$
d_{i}^{j}=\widehat{d_{1}^{j}}+d_{\epsilon}^{j},
$$

where $\widehat{d_{1}^{j}}$ and $d_{\epsilon}^{j}$ denote the disparity compensated wavelet coefficients in the first frame and the wavelet coefficients of the $\alpha$ Lipschitz error, respectively. By definition, the wavelet coefficients of the $\alpha$-Lipschitz error can be upper bounded as:

$$
\left|d_{\epsilon}^{j}\right| \leq A_{\epsilon} 2^{-j(\alpha+1)} .
$$

Referring to (3), this analysis can be used to determine the number of LSB which must be transmitted to correctly reconstruct the texture. A similar analysis can be applied when the subbands of the signal are quantized.

The RD function of a globally smooth 2D $\alpha$-Lipschitz signal, which is encoded using a DSC scheme can be shown to have the same RD behaviour as in (7). Therefore, the total RD due to encoding the dataset at the server using the DSC scheme is identical to (10) with different scaling constants. This behaviour will be validated in the following section. 


\section{SIMULATION RESULTS AND ALGORITHM ANALYSIS}

To evaluate the proposed algorithm, we compare the RD performance of our method to JPEG2000. The independent coding scheme has been modified to include the same entropy coding as our algorithm. We first show that the proposed method achieves the optimal $\mathrm{RD}$ performance presented in Section 4.3 for the signal model of Section 4.1. We then present numerical results on real data.

To this end, we encode an $\alpha=1.5$ Lipschitz multiview image array consisting of four images. The data is encoded using a linear compression strategy where the optimal rate allocation for each image is obtained using (12). The model parameters are estimated by separately encoding each image in DSC or independent mode. Fig. 5 shows the theoretical and practical results when encoding the

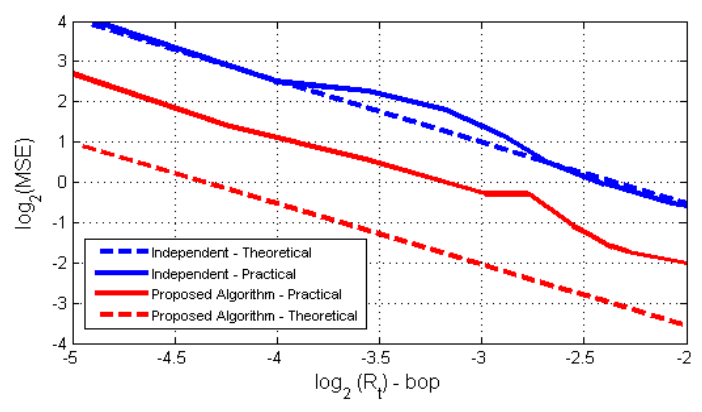

Fig. 5. Practical and theoretical RD performance when encoding an $\alpha=1.5$ Lipschitz signal. The proposed algorithm achieves an improved performance in both practical and theoretical cases. Observe that the rate of decay in both the theoretical and practical cases is the same.

$\alpha$-Lipschitz signal. Observe that the performance of the proposed algorithm is better in both the theoretical and practical results. As conjectured in Section 4.3, the independent and proposed algorithms have the same rate of decay but with different scaling constants.

We use the data sequence called 'Tsukuba Light Field' $(272 \times$ $368 \times 4 \times 4)$ from [12]. The first image is transmitted using the intra modality. Then, to mimic random access, the other images are randomly chosen and DSC encoded using our algorithm. To obtain the RD curve below, the data is encoded once at the server and decoded multiple times by setting different values of the variable $\lambda$. In practice, the correct value can be obtained using a training set or estimated online while the data set is decoded. In the independent case, each point is optimized at the server and represents the optimal RD performance. Fig. 6 shows the RD performance of the

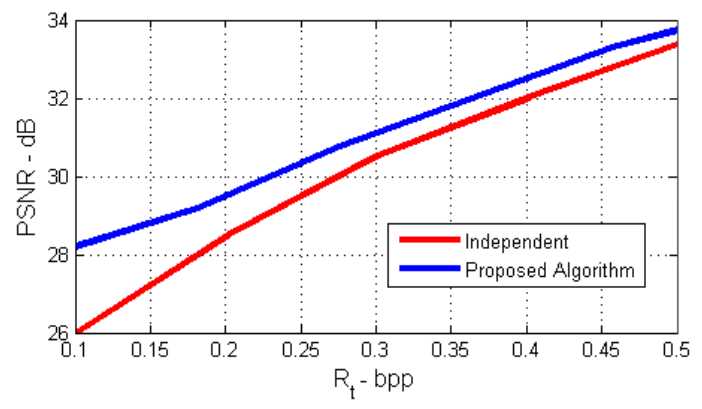

Fig. 6. RD performance of the proposed algorithm and JPEG2000 when encoding 'Tsukuba Light Field'.

proposed algorithm in comparison to JPEG2000. Observe that our approach consistently outperforms the independent coding scheme and achieves a gain of $1.6 \mathrm{~dB}$ at $0.15 \mathrm{bpp}$. Due to a lack of space, we only show experimental results for one real dataset. However, similar results are observed on other data sequences.

\section{CONCLUSION}

We presented a novel multiview image compression algorithm with random access at image level. The fundamental component of the algorithm is the layer-based representation, which partitions the data into redundant layers each modeled by a constant depth value. Each layer is encoded independently and we use robust DSC coding principles to remove the side information ambiguity at the decoder. The algorithm achieves an improved RD performance with gains of up to 1.6dB over independent encoding. Furthermore, we have presented a RD analysis of our algorithm which demonstrates that the proposed approach can achieve a better performance in comparison to independent coding.

\section{REFERENCES}

[1] C. Zhang and T. Chen, "A survey on image-based renderingrepresentation, sampling and compression," Signal Processing: Image Communication, vol. 19, no. 1, pp. 1-28, 2004.

[2] M. Magnor and B. Girod, "Data compression for light-field rendering," IEEE Transactions on Circuits and Systems for Video Technology, vol. 10, no. 3, pp. 338-343, Apr 2000.

[3] B. Girod, C.L. Chang, P. Ramanathan, and X. Zhu, "Light field compression using disparity-compensated lifting," IEEE Transactions on Image Processing, pp. 761-764, 2003.

[4] A. Gelman, P.L. Dragotti, and V. Velisavljevic, "Multiview image coding using depth layers and an optimized bit allocation," submitted to IEEE Transactions on Image Processing.

[5] P. Ramanathan and B. Girod, "Random access for compressed light fields using multiple representations," in Multimedia Signal Processing, 2004 IEEE 6th Workshop on, 2004.

[6] N.-M. Cheung, A. Ortega, and G. Cheung, "Distributed source coding techniques for interactive multiview video streaming," in Proceedings of the 27th conference on Picture Coding Symposium, Piscataway, NJ, USA, 2009, PCS'09, pp. 269-272, IEEE Press.

[7] A. Aaron, P. Ramanathan, and B. Girod, "Wyner-ziv coding of light fields for random access," in Multimedia Signal Processing, 2004 IEEE 6th Workshop on, 2004.

[8] J. Berent and P.L. Dragotti, "Plenoptic manifolds: Exploiting structure and coherence in multiview images," IEEE Signal Processing Magazine, vol. 24, no. 6, pp. 34-44, November 2007.

[9] S. Li and W. Li, "Shape-adaptive discrete wavelet transforms for arbitrarily shaped visual object coding," IEEE Transactions on Circuits and Systems for Video Technology, vol. 10, no. 5, pp. 725-743, Aug 2000.

[10] D. Taubman, "High performance scalable image compression with EBCOT," IEEE Transactions on Image Processing, vol. 9, pp. 1158$1170,2000$.

[11] S. Mallat, A Wavelet Tour of Signal Processing, Third Edition: The Sparse Way, Academic Press, 3rd edition, 2008.

[12] D. Scharstein and R. Szeliski, "Middlebury data sets," vision.middlebury.edu/stereo/. 